\title{
Les stratégies du courrier électronique
}

\section{Annick Schott}

\section{OpenEdition \\ Journals}

Édition électronique

URL : https://journals.openedition.org/communicationorganisation/2459

DOI : 10.4000/communicationorganisation.2459

ISSN : $1775-3546$

\section{Éditeur}

Presses universitaires de Bordeaux

\section{Édition imprimée}

Date de publication : 1 novembre 2000

ISSN : 1168-5549

\section{Référence électronique}

Annick Schott, "Les stratégies du courrier électronique », Communication et organisation [En ligne], 18 | 2000, mis en ligne le 27 mars 2012, consulté le 05 août 2021. URL : http://journals.openedition.org/ communicationorganisation/2459; DOI : https://doi.org/10.4000/communicationorganisation.2459

Ce document a été généré automatiquement le 5 août 2021

(C) Presses universitaires de Bordeaux 


\title{
Les stratégies du courrier électronique
}

\author{
Annick Schott
}

\section{NOTE DE L'ÉDITEUR}

Les annexes seront fournies sur demande. Pour ce faire contacter Annick Schott :

schott@montaigne.u-bordeaux.fr

1 Malgré l'apparente ambiguïté d'un tel titre pour une telle communication, nous allons tenter de confirmer ou d'infirmer l'hypothèse selon laquelle l'utilisation du mail en entreprise aurait tendance à aussi formaliser une partie de la communication nonverbale.

2 Parce qu'elles permettent une circulation d'informations de plus en plus volumineuses et rapides, les technologies de l'information génèrent de plus en plus de transparence dans l'entreprise. L'activité peut être traitée par projets. Ainsi il est aisé de mesurer ce que rapporte chacun dans la chaîne des valeurs. Chaque acteur devient transparent. Cette transparence humaine accompagne l'utilisation des mails. Non seulement ces derniers donnent à n'importe qui la possibilité d'accéder à n'importe quelle strate hiérarchique de l'entreprise («transparence » organisationnelle), mais ils véhiculent les tensions que génèrent les réponses face aux exigences inter-services ou externes ( « transparence » émotionnelle).

3 À ce propos, pourquoi avoir choisi les pratiques du courrier électronique en entreprise et la communication non-verbale? Quel procédé utiliser pour confirmer ou infirmer une telle hypothèse ? Cette première question est destinée à aborder notre champ théorique, la seconde, notre expérimentation. 


\section{Cadre théorique}

4 Il nous faut présenter le pourquoi du courrier électronique et le pourquoi de l'entreprise.

\section{Pourquoi le courrier électronique?}

5 Roger Chartier ${ }^{1}$ parle à propos des médias électroniques du livre en révolution. Le courrier électronique permet en effet de saisir un maximum d'éléments en un minimum de temps. Il permet donc aussi une lecture condensée de prélèvement d'informations. En entreprise son usage s'ancre dans la réalité sociale, empreinte de subjectivité.

6 En effet, cette réalité sociale, c'est l'action sociale dans laquelle chaque individu au travail est engagé de telle façon que ce phénomène soit partagé par tous, mais au sein duquel chacun peut en avoir une expérience différente. Cependant, de nombreuses expériences, notamment celles d'Elton Mayo ont montré qu'un groupe tend à produire une homogénéité de valeurs et de normes. Ainsi, lorsqu'un individu veut que ses écrits soient admis auprès de groupe, il va ajuster son écriture autour de la norme du groupe sous peine de non acceptation de son action. C'est la sanction institutionnelle de la nonconformité. Ainsi conformité, normes constituent un lien entre l'action d'un individu et la structure sociale. Qui dit intégration de l'action d'un acteur dans un groupe dit ajustement mutuel.

\section{Pourquoi l'entreprise?}

Bruno Lussoto ${ }^{2}$ dans son ouvrage Introduction critique aux théories des organisations élabore une partition de l'information d'entreprise en trois zones (zone quantifiée et quantifiable. zone qualitative fortement formalisée, zone informelle). Ces zones « regroupent les processus, les activités, les décisions, les opérations et les problèmes " rangés par degré de formalisation croissante. Ce qui nous intéresse, figure dans la zone informelle soit « des gestes, des mimiques des sous-entendus, voire des allusions, "... des attitudes affectives ou passionnelles. Cette logique des sentiments approfondie par Jean-Daniel Reynaud ${ }^{3}$ comprend les réseaux d'amitiés, les traditions professionnelles, les relations interpersonnelles et leur histoire, l'honneur et les rapports de prestige et. " en dépit de son aspect effacé, joue un rôle considérable dans les opérations de choix et de décisions ».

Certaines de ces attitudes sont recherchées par l'organisation. Ainsi l'humour aurait le pouvoir de fédérer et de déstresser ${ }^{4}$. À l'intérieur de l'entreprise, le mail exerce-t-il un rôle similaire au même titre que certains phénomènes d'ambiance?

\section{L'expérimentation}

Destinée à confirmer, infirmer ou réécrire notre hypothèse, cette recherche se devait de respecter les étapes incontournables de la construction et du choix du corpus, et de la méthode d'analyse et de l'analyse des résultats. 


\section{Construction et choix du corpus}

10 Nous avons choisi d'étudier les mails de trois organisations différentes : une collectivité locale, une start-up et un EPIC ${ }^{5}$.

11 Bien sûr seuls des liens de confiance pouvaient nous permettre d'accéder à des mails dont l'objet matérialise des relations de travail entre collaborateurs et supérieurs hiérarchiques. L'accès à ce type de document est fermé, autant que peut l'être un compte rendu de réunion de travail d'une équipe intra ou inter service ou même le P.V. d'une quelconque instance représentative du personnel d'une quelconque entreprise. Tous ces éléments vers l'extérieur ne s'affichent pas et sont parfois classés confidentiels. Cette confiance totale auprès de nos contacts interpersonnels était donc plus qu'indispensable. Cette étude est donc originale. À notre connaissance, d'autres études de ce type n'ont pas été menées. Les premières recherches expérimentales qui peuvent se rapprocher de notre étude concernent les échanges sur le web. Le volume XVII de la revue Réseaux, intitulé "Internet, un nouveau mode de communication ", publié en 1999, n 97 chez Hermes, analyse la relation entre l'écriture des messageries électroniques et l'existence d'un lien social, ainsi que celle des forums de discussion avec l'affirmation d'une identité sociale.

Il s'agit d'échanges à caractère privé et personnel mis sur le réseau public. Alors que notre étude ne s'intéresse qu'à des échanges de travail et professionnels véhiculés par le réseau privé d'une entreprise ou d'un organisme.

Ainsi, les mails étudiés émanent de cadres dirigeants ou supérieurs vers leurs homologues et leurs collaborateurs et inversement d'homologues et de collaborateurs vers leurs cadres dirigeants ou supérieurs.

\section{Pourquoi ces types d'organisation?}

14 À partir de la typologie organisationnelle d'Henry Mintzberg ${ }^{6}$, nous pouvons considérer que les trois organisations choisies appartiennent à des modèles différents.

15 Pour la collectivité locale et l'EPIC, ce sont plutôt « la standardisation des procédés, la spécialisation des postes " à qualification moyenne qui prévalent (modèle de bureaucratie mécaniste); teintée cependant pour l'EPIC de certaines caractéristiques de la bureaucratie professionnelle (compétences et métier) et pour la collectivité locale de normes et des croyances apparentées à l'organisation missionnaire.

16 La start-up organise la concordance entre les différentes tâches autour de la transversalité d'équipes hautement qualifiées. Il s'agit à première vue d'un modèle d'adhocratie ou organisation innovatrice, puisque dans cette structure fluide «la coordination s'opère par ajustement mutuel au moyen de communication informelle ».

17 On le perçoit par leur type d'organisation, la communication pour ces trois structures ne revêt pas la même importance.

18 Pour la start-up faire émerger de nouvelles solutions, évaluer leur valeur ajoutée, identifier leurs auteurs, les inciter à innover par un système de récompenses au plus juste,... est une question de « survie ». D'autant que « une fois parvenue au succès, ces entreprises perdent la fibre de l'innovation, deviennent comme les autres, la créativité se trouve contrariée par le poids des habitudes,... la distribution classique des pouvoirs et des responsabilités ${ }^{7} »$. Comment dans ces conditions se consacrer entièrement à 
l'innovation, comment retrouver l'esprit start-up ? Certaines d'entre elles voient en la communication une des solutions à « la prise d'un second souffle... Il s'agit de pouvoir s'adresser quelque part dans l'entreprise à quelqu'un qui apportera son aide ».

Pour une collectivité locale, le pouvoir de communiquer permet de justifier voire même de sublimer certaines des actions, parfois politiques, du Maire et de l'équipe municipale. Selon Philippe Breton et Serge Proul ${ }^{8}$. «plusieurs facteurs convergents expliquent ce mariage.... entre politique et communication... notamment parce que la démocratie transforme le citoyen en cible vivante d'une argumentation politique visant à le convaincre d'adhérer à telle ou à telle cause ». Cependant, la désignation d'un Maire et de son équipe municipale obéit à des règles spécifiques au cœur desquelles dominent personnalisation, affectivité et sentiments. La logique des sentiments et la communication informelle qui s'y rattache revêt là encore tout son importance.

L'EPIC, malgré ou à cause de son statut d'entreprise de service public chargée des transports ferroviaires, a notamment développé depuis plus de 10 ans ses capacités et sa volonté d'écoute. La priorité des priorités est le voyageur. La satisfaction-client ordonnance les capacités d'évolution et d'adaptation de l'entreprise. Bien communiquer avec le client devient un enjeu. Un enjeu à faire partager aux agents. L'utilisation active et canalisée de la communication interne doit marier la mise en œuvre des orientations générales de l'entreprise avec la sollicitation des agents confrontées aux réalités locales. Comment alors opinions et tensions s'exprimentelles? Le mail joue-t-il un rôle?

\section{Pourquoi ce type de mail ?}

21 Pour répondre à nos préoccupations nous avons choisi un corpus d'écrits électroniques. Pour parvenir à effectuer un choix plusieurs paramètres ont été pris en compte Ces écrits concernent l'activité professionnelle. Ils s'inscrivent dans des rapports de communication transversale, ascendante. Sont identifiés le qui parle à qui, le moment et le lieu de l'échange, ainsi que l'historique de l'événement Les courriers électroniques ont été sélectionnés par les destinataires, qui nous les ont confiés.

\section{Méthode d'analyse et analyse des résultats.}

Nous avions besoin d'un outil d'analyse qui, non seulement nous permette de vérifier notre hypothèse, mais également nous donne un moyen de mettre de la distance par rapport à nos présupposés. L'analyse de contenu'constitue une méthode qui correspond à nos attentes. Elle cherche à savoir ce qui est derrière les paroles sur lesquelles elle se penche. Elle évite de recourir à l'intuition aux impressions personnelles et permet d'éliminer la subjectivité.

\section{Méthode d'analyse}

23 L'analyse de contenu n'est pas que quantitative. A.L. George ${ }^{10}$ soulignait en 1959 qu'elle pouvait être qualitative. Elle se caractérise alors par l'inférence fondée sur la présence de l'indice retenu (exemple présence d'un thème, d'éléments expressifs indiquant l'ironie ou le courroux, etc.). Elle est valable pour faire des déductions spécifiques à propos d'un événement précis. Elle peut fonctionner sur un corpus réduit. L'analyse qualitative comporte des risques, celui de laisser des éléments importants ou de 
prendre en compte des éléments non significatifs. La prise en compte des conditions de production du message permet de limiter ce type de risques. Ainsi dans le corpus de mails étudiés, nous savons qui parle à qui, dans quelles circonstances, à quel moment, dans quel lieu, et nous disposons de l'historique de l'événement.

Pour permettre une lecture rigoureuse des mails reçus, nous avons découpé le texte par thème et sous thème. Ce qui nous intéresse, ce n'est pas la fréquence d'occupation de telle ou telle thématique par phrase ou groupe de phrases, mais la présence ou non d'indices relatifs à la manifestation de l'émotion dans des échanges par mail d'informations opérationnelles. Nous avons donc choisi de fonder l'analyse des mails sur le schéma de communication de Roman Jakobson.

«Le destinateur envoie un message au destinataire. Celui-ci peut orienter son message vers la révélation de son état subjectif (Fonction Expressive ou Emotive). Il peut aussi vouloir poursuive comme objectif d'imposer une raison ou plutôt La raison pour laquelle et à laquelle doit se rallier le destinataire (Fonction Conative). Il peut souhaiter s'attacher à l'esthétique du message, sa préoccupation stylistique, sa forme créatrice de sens (Fonction Poétique). Pour être opérant le message requiert un contexte auquel il renvoie...... contexte saisissable par le destinataire et qui est soit verbal, soit susceptible d'être verbalisé (Fonction Référentielle); le message requiert un code, commun en tout ou au moins en partie, au destinateur et au destinataire... (Fonction Métalinguistique); enfin, le message requiert un contact, un canal physique et une connexion psychologique entre le destinateur et le destinataire, contact qui permet de maintenir la communication (Fonction Phatique) ».

A l'aide de cette classification, il faudra qualifier la structure du texte en fonction de la présence de la fonction dominante et d'une ou plusieurs fonctions secondaires. Ce qui en final peut donner une formule tel $\mathrm{E}(\mathrm{RC}) \mathrm{E}$ = fonction expressive ou émotive qui domine le texte, $(\mathrm{RC})$ sont les fonctions secondaires. Dans ce cas le texte offre à son destinataire de partager l'émotion de l'émetteur. Cette émotion a un contexte et s'appuie sur quelques discrètes raisons.

Chaque mail a fait l'objet d'une lecture à l'aide d'une grille d'analyse. Nous avons conçu la grille d'analyse pour qu'à chaque thème et sous thème soient prises en compte les trois fonctions qui nous considérons comme essentielles dans l'analyse proposée par Jakobson, c'est-à-dire la fonction référentielle, la fonction émotive et la fonction conative. La fonction référentielle induit des modalités de neutralité, la fonction émotive des modalités de réactions (positives ou négatives) la fonction conative des modalités d'initiative (d'idée ou concrètes). 


\section{Analyse des résultats} catégorie, 3 émanent de commerciaux ${ }^{13}$ Les commerciaux ont aussi pour objectif d'orienter leur communication vers un changement d'attitudes ou de comportement de leur destinataire (fonction conative). Sur les 3 mails qui entrent dans cette catégorie, 2 émanent de commerciaux ${ }^{14}$ L'intégralité des mails s'adresse à un homologue des services financiers ou comptables. Dans tous les cas, lorsque la fonction émotive est dominante, la fonction conative joue les seconds rôles (mails $n^{\circ}$ VIII, XI et XII) et inversement, lorsque la fonction conative est dominante, la fonction émotive joue les seconds rôles (mails $n^{\circ}$ VI et IX). Les références aux faits sont accessoires. Ce qui compte, c'est faire partager son urgence (émotive ou conative) pour faire accepter des 
changements de priorité quitte à bouleverser les habitudes pour satisfaire au mieux le client.

\section{Conclusion}

S'il est vrai que la logique des sentiments existe dans cette start-up, la révéler à partir de 13 mails reste insuffisant.

Cependant la pratique de la bonne traitance est autorisée dans les rapports entre les services étudiés. Nous pensons plus particulièrement à la direction financière. En effet nous avons pu, à plusieurs reprises, observer le comportement du directeur financier avec ses subordonnés directs et indirects. Ils sont plus que civiques, cordiaux. Et pourtant, les pressions de rentabilité pèsent sur les épaules de ce cadre dirigeant. Mais, il se défend, par son attitude, de faire monter la pression au détriment de ses salariés pour le bien des intérêts de l'entreprise.

D'autres pistes de recherche restent encore à exploiter, plus visibles peut-être tels le comportement incivique et la maltraitance autorisée pour atteinte des résultas objectivés par la direction de l'entreprise...

\section{BIBLIOGRAPHIE}

BARDIN L., L'analyse de contenu, Paris, PUF, coll. le psychologue, 1977, 233 p.

BRETON P., Proulx S., L'Explosion de la communication, Paris. La Découverte/Boréal, coll. Sciences et société, 1989, 247 p. Chartier R., Le livre en révolution, entretien avec Jean Lebrun, Paris, Ed. Textuel, 1997, 159 p.

JAKOBSON R., Essais de linguistique générale, Paris, Ed. du Seuil, 1970, 255 P-

LUSSOTO B., Introduction critique aux théories des organisations. Paris, Ed. Dunod, coll. Systémique, réédition, 1998, $250 \mathrm{p}$.

MINTZBERG H.. Structure et dynamique des organisations, Paris, Ed. d'Organisation, 1982, 360 p.

REYNAUD J-D., Les règles du jeu. L'action collective et la régulation sociale, Paris, Armand Colin, réédition, 1997, $420 \mathrm{p}$.

ROBERT A., Bouillaguet A., L'analyse de contenu. Paris, PUF, coll. Que sais-je ?, 1997126 p.

\section{NOTES}

1. Roger Chartier, Le livre en révolution, entretien avec Jean Lebrun, Paris, Ed. Textuel, 1997, 159 p.

2. Bruno Lussoto, Introduction critique aux théories des organisations, Paris, Ed. Dunod, coll. Systémique. réédition, 1998. p.

3. Jean-Daniel Reynaud, Les règles du jeu. L'action collective et la régulation sociale, Paris, Armand Colin, réédition. 1997. 420 p. 
4. C'est le sens de l'article de Laurance N'kaoua et d'Agnès Laurent, «l'entreprise, ça vous fait vraiment rire? » publié dans L'Usine Nouvelle n² 2681 du 1er avril 1999 p. 26.

5. Etablissement Public à caractère Industriel et Commercial.

6. Henry Mintzberg, Structure et dynamique des organisations, Paris, Ed. d'Organisation, 1982, $360 \mathrm{p}$.

7. Etude confidentielle de l'association Croissance Plus publiée dans les pages initiatives Le Monde du 13 avril 1999

8. Philippe Breton et Serge Proulx, L'Explosion de la communication, Paris, La Découverte/Boréal, coll. Sciences et société, 1989, 247 p.

9. "c'est un ensemble de techniques d'analyse de communication visant... à obtenir des indicateurs (quantitatifs ou non) permettant I inference de connaissances relatives aux conditions de production/ réception... de ces messages" selon André Robert et Annick Bouillaguet, L 'analyse de contenu, Paris, PUF, coll. Que sais-je 7, 1997126 p.

10. A.L. George "Quantitative and qualitative approaches to content analysis » p. 115 in Laurence Bardin, L analyse de contenu, Paris, PUF, coll. le psychologue, 1977.

11. Roman Jakobson, Essais de linguistique générale, Paris, Ed. du Seuil, 1970, 255

12. cf. annexe mails IV, $X$

13. cf. annexe mails VIII, XI, XII

14. cf. annexe mails VI, IX

\section{AUTEUR}

\section{ANNICK SCHOTT}

Université de Bordeaux 3 